\title{
Transient MHD Natural Convection Between Two Vertical Walls Heated/Cooled Asymmetrically
}

\author{
B. C. Sarkar \\ Department of Applied Mathematics, \\ Vidyasagar University \\ Midnapore 721 102, India
}

\author{
S. Das \\ Department of Mathematics, \\ University of Gour Banga \\ Malda 732 103, India
}

\author{
R. N. Jana \\ Department of Applied Mathematics, \\ Vidyasagar University, \\ Midnapore 721 102, India
}

\begin{abstract}
The transient MHD natural convection flow of a viscous incompressible electrically conducting fluid confined between vertical walls heated/cooled asymmetrically has been studied. We have considered two different cases (i) when one of the walls is stationary and (ii) when one of the walls starts to move impulsively. The governing equations have been solved analytically using the Laplace transform technique. The velocity field and temperature distribution are being presented graphically. The fluid velocity decreases for both the stationary wall as well as for impulsive motion of one of the walls with an increase in either magnetic parameter or Prandtl number. An increase in fluid temperature occurs due to an increase in temperature difference ratio. Further, the shear stress increases for both the stationary wall as well as for impulsive motion of one of the walls with an increase in either Grashof number or temperature difference ratio or time. The rate of heat transfer at the wall $\eta=0$ increases with an increase in Prandtl number while it decreases with an increase in temperature difference ratio.
\end{abstract}

Keywords: Transient MHD natural convection, Prandtl number, Grashof number, temperature difference ratio, stationary wall, impulsive motion and rate of heat transfer.

\section{INTRODUCTION}

MHD flows with heat transfer in electrically conducting fluid have attracted substantial interest in the context of metallurgical fluid dynamics, re-entry aerothermodynamics, astronautics, geophysics, nuclear engineering and applied mathematics. Investigation of the MHD natural convection transport processes due to the coupling of the fluid flow and heat transfer is a challenging as well as interesting phenomenon. It has been extensively studied between vertical walls because of its importance in many engineering applications in the fields of nuclear reactors, heat exchangers, cooling appliances in electronic instruments. These flows have been studied by assuming the plates at two different constant temperatures or temperature of the plates varying linearly along the plates etc. Singer (1965) has assessed the unsteady free convection heat transfer with magnetohydrodynamic effects in a channel regime. Joshi (1988) has studied the transient effects in natural convection cooling of vertical parallel plates. Singh (1988) has investigated the effect of free convection in unsteady Couette motion between two vertical parallel plates. Sacheti et al. (1994) have discussed an exact solution for unsteady magnetohydrodynamic free convection flow with constant heat flux. The transient free convection flow between two vertical parallel plates has been investigated by Singh et al.(1996). An exact solution for transient free convection MHD Couette flow with impulsive motion of one of the plates has been discussed by
Jha (2001). Chamkha (2001) has studied the unsteady laminar hydromagnetic flow and heat transfer in porous channel with temperature-dependent properties and inertial effects on the convection regime. Jha et al. (2003) have analyzed the transient free convection flow in a vertical channel due to symmetric heating. Singh and Paul (2006) have described the transient natural convection between two vertical walls heated/cooled asymmetrically. Thermal radiation effect on fully developed mixed convection flow in a vertical channel has been studied by Grosan and Pop (2007). The natural convection in unsteady Couette flow between two vertical parallel plates in the presence of constant heat flux and radiation has been presented by Narahari (2009). Narahari (2009) has presented an exact solution to the problem of unsteady free convective flow of dissipative viscous incompressible fluid between two long vertical parallel plates in which the temperature of one of the plates is oscillatory where as the temperature of the other plate is uniform. Narahari (2010) has investigated the effects of thermal radiation and free convection currents on the unsteady Couette flow between two vertical parallel plates with constant heat flux at one boundary. Rajput and Pradeep (2011) have studied the effect of a uniform transverse magnetic field on the unsteady transient free convection flow of an incompressible viscous electrically conducting fluid between two infinite vertical parallel plates with constant temperature and Variable mass diffusion. Das et al.(2012) have studied the radiation effects on free convection MHD Couette flow started exponentially with variable wall temperature in presence of heat generation. Effect of radiation on transient natural convection flow between two vertical walls has been investigated by Mandal et al. (2012). Ahmed et al. (2012) have presented the magnetic field effect on an unsteady free convection flow of a viscous incompressible electrically conducting fluid with dissipative heat between two long vertical parallel plates where the temperature of one of the plates oscillates about a constant non-zero mean temperature. Recently, Sarkar et al.(2012) have investigated the effects of radiation on MHD free convective couette flow in a rotating system.

In this paper, we study the transient MHD natural convection flow of a viscous incompressible electrically conducting fluid confined between two infinite vertical walls heated/cooled asymmetrically. It is observed that the velocity $u_{1}(\eta)$ decreases for both stationary walls as well as for impulsive motion of one of the walls with an increase in either magnetic parameter $M^{2}$ or Prandtl number $\mathrm{Pr}$. It is also observed that the velocity $u_{1}(\eta)$ increases with an increase in either Grashof number $\mathrm{Gr}$ or temperature difference ratio $r_{T}$ or time $\tau$ for both stationary wall as well as for impulsive motion of one of the walls. The effect of the Prandtl number $P r$ is very important in the temperature field. An increase in temperature $\theta(\eta)$ occurs due to an increase in either time or temperature difference ratio. 
Further, the shear stress $\tau_{x}$ at the wall $\eta=0$ increases for both stationary wall as well as for impulsive motion of one of the walls with an increase in either Grashof number $\mathrm{Gr}$ or temperature difference ratio $r_{T}$ or time $\tau$. The rate of heat transfer $-\theta^{\prime}(0)$ at the wall $\eta=0$ increases with an increase in Prandtl number $\mathrm{Pr}$.

\section{FORMULATION OF THE PROBLEM AND ITS SOLUTIONS}

Consider the unsteady natural convective flow of a viscous incompressible electrically conducting fluid between two infinite vertical parallel walls separated by a distance $h$ in the presence of a transverse magnetic field. Choose a cartesian co-ordinates system with the $x$-axis along one of the walls in the vertically upward direction and the $y$-axis normal to the walls [See Fig.1]. Initially, at time $t \leq 0$, the two walls and the fluid are assumed to be at the same temperature $T_{m}$ and stationary. At time $t>0$, the wall at $y=0$ starts moving in its own plane with a velocity $\lambda u_{0}$ and it is heated with temperature $T_{0}$ whereas the wall at $y=h$ is maintained at a constant temperature $T_{h}$. A uniform magnetic field of strength $B_{0}$ is imposed perpendicular to the walls. As the walls are infinitely long, the velocity and temperature fields are functions of $y$ and $t$ only.

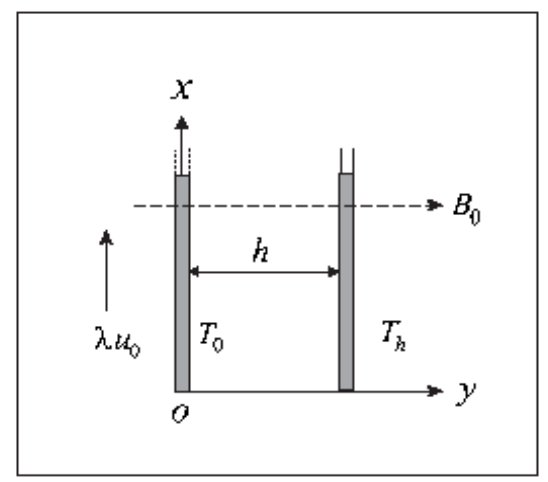

Figure 1 : Geometry of the problem

Under the usual Boussinesq's approximation, the fluid flow be governed by the following system of equations:

$$
\begin{gathered}
\frac{\partial u}{\partial t}=v \frac{\partial^{2} u}{\partial y^{2}}+g \beta^{*}\left(T-T_{m}\right)-\frac{\sigma B_{0}^{2}}{\rho} u, \\
\rho c_{p} \frac{\partial T}{\partial t}=k \frac{\partial^{2} T}{\partial y^{2}},
\end{gathered}
$$

where $u$ is the velocity in the $x$-direction, $g$ the acceleration due to gravity, $T$ the fluid temperature, $T_{m}$ the initial fluid temperature, $\beta^{*}$ the coefficient of thermal expansion, $v$ the kinematic coefficient of viscosity, $\rho$ the fluid density, $\sigma$ the electric conductivity, $k$ the thermal conductivity and $c_{p}$ the specific heat at constant pressure.

The initial and the boundary conditions for velocity and temperature distributions are as follows:

$$
\begin{aligned}
& u=0, T=T_{m} \text { for } 0 \leq y \leq h \text { and } t \leq 0, \\
& u=\lambda u_{0}, T=T_{0} \text { at } y=0 \text { for } t>0, \\
& u=0, T=T_{h} \text { at } y=h \text { for } t>0,
\end{aligned}
$$

where $(\lambda=0)$ for stationary wall and $(\lambda=1)$ when the wall at $(y=0)$ starts to move impulsively.

We introduce non-dimensional variables

$$
\eta=\frac{y}{h}, \tau=\frac{v t}{h^{2}}, u_{1}=\frac{u}{u_{0}}, \theta=\frac{T-T_{m}}{T_{0}-T_{m}} .
$$

On the use of (4), equations (1) and (2) become

$$
\begin{aligned}
& \frac{\partial u_{1}}{\partial \tau}=\frac{\partial^{2} u_{1}}{\partial \eta^{2}}+G r \theta-M^{2} u_{1}, \\
& \frac{\partial \theta}{\partial \tau}=\frac{1}{\operatorname{Pr}} \frac{\partial^{2} \theta}{\partial \eta^{2}},
\end{aligned}
$$

where $M^{2}=\frac{\sigma B_{0}^{2} h^{2}}{\rho v}$ is the magnetic parameter, $\operatorname{Pr}=\frac{\rho v c_{p}}{k}$ the Prandtl number and $G r=\frac{g \beta^{*}\left(T_{0}-T_{m}\right) h^{2}}{v u_{0}}$ the Grashof number.

The corresponding initial and boundary conditions for $u_{1}$ and $\theta$ are

$$
\begin{aligned}
& u_{1}=0, \theta=0 \text { for } \varangle \eta \leq 1 \text { an } \pi \leq \\
& u_{1}=\lambda, \theta=1 \text { at } \eta=0 \text { for } \tau> \\
& u_{1}=0, \theta=r_{T} \text { at } \eta=1 \text { fo }>
\end{aligned}
$$

where $r_{T}=\frac{T_{h}-T_{m}}{T_{0}-T_{m}}$ is the wall temperature difference ratio.

Taking Laplace transformation of the equations (5) and (6), we get

$$
\begin{aligned}
& \frac{d^{2} \bar{u}_{1}}{d \eta^{2}}-\left(M^{2}+s\right) \bar{u}_{1}=-G r \bar{\theta}, \\
& \frac{d^{2} \bar{\theta}}{d \eta^{2}}=s P \bar{\theta},
\end{aligned}
$$

where

$$
\bar{u}_{1}(\eta, s)=\int_{0}^{\infty} u_{1}(\eta, \tau) e^{-s \tau} d \tau \text { and } \bar{\theta}(\eta, s)=\int_{0}^{\infty} \theta(\eta, \tau) e^{-s \tau} d \tau .
$$

The corresponding boundary conditions for $\bar{u}_{1}$ and $\bar{\theta}$ are

$$
\begin{aligned}
& \bar{u}_{1}(0, s)=\frac{\lambda}{s}, \bar{\theta} \quad(0,=) \frac{1}{s} \\
& \bar{u}_{1}(1, s)=0 \bar{\theta} \quad\left(s,=\frac{r_{T}}{s}\right.
\end{aligned}
$$

The solution of the equations (8) and (9) subject to the boundary conditions (11) are easily obtained and given by

$$
\bar{\theta}(\eta, s)= \begin{cases}\frac{1}{s}\left[\frac{\sinh \sqrt{\operatorname{Prs}}(1-\eta)}{\sinh \sqrt{\operatorname{Pr} s}}+r_{T} \frac{\sinh \sqrt{\operatorname{Prs}} \eta}{\sinh \sqrt{\operatorname{Pr} s}}\right] & \text { for } \operatorname{Pr} \neq 1 \\ \frac{1}{s}\left[\frac{\sinh \sqrt{s}(1-\eta)}{\sinh \sqrt{s}}+r_{T} \frac{\sinh \sqrt{s} \eta}{\sinh \sqrt{s}}\right] & \text { for } \operatorname{Pr}=1,\end{cases}
$$




$$
\bar{u}_{1}(\eta, s)=\left\{\begin{array}{l}
\frac{\lambda}{s} \frac{\sinh \sqrt{M^{2}+s}(1-\eta)}{\sinh \sqrt{M^{2}+s}} \\
+\frac{G r}{(\operatorname{Pr}-1) s(s-\beta)}\left[\left\{\frac{\sinh \sqrt{M^{2}+s}(1-\eta)}{\sinh \sqrt{M^{2}+s}}+r_{T} \frac{\sinh \sqrt{M^{2}+s} \eta}{\sinh \sqrt{M^{2}+s}}\right\}\right. \\
\left.-\left\{\frac{\sinh \sqrt{\operatorname{Pr} s}(1-\eta)}{\sinh \sqrt{\operatorname{Pr} s}}+r_{T} \frac{\sinh \sqrt{\operatorname{Pr} s} \eta}{\sinh \sqrt{\operatorname{Prs}}}\right\}\right] \\
\frac{\lambda}{s} \frac{\sinh \sqrt{M^{2}+s}(1-\eta)}{\sinh \sqrt{M^{2}+s}}-\frac{G r}{M^{2} s}\left[\left\{\frac{\sinh \sqrt{M^{2}+s}(1-\eta)}{\sinh \sqrt{M^{2}+s}}+r_{T} \frac{\sinh \sqrt{M^{2}+s} \eta}{\sinh \sqrt{M^{2}+s}}\right\}\right. \\
\left.-\left\{\frac{\sinh \sqrt{s}(1-\eta)}{\sinh \sqrt{s}}+r_{T} \frac{\sinh \sqrt{s} \eta}{\sinh \sqrt{s}}\right\}\right]
\end{array}\right.
$$

where $\beta=\frac{M^{2}}{P r-1}$.

The inverse Laplace transforms of (12) and (13) give the solutions for the temperature distribution and velocity field as

$$
\begin{aligned}
& \theta(\eta, \tau)= \begin{cases}\sum_{n=0}^{\infty}\left[F_{1}\left(a_{1} \sqrt{P r}, \tau\right)-F_{1}\left(a_{2} \sqrt{P r}, \tau\right)\right. & \\
\left.+r_{T}\left\{F_{1}\left(a_{3} \sqrt{\operatorname{Pr}}, \tau\right)-F_{1}\left(a_{4} \sqrt{\operatorname{Pr}}, \tau\right)\right\}\right] & \text { for } \operatorname{Pr} \neq 1 \\
\sum_{n=0}^{\infty}\left[F_{1}\left(a_{1}, \tau\right)-F_{1}\left(a_{2}, \tau\right)+r_{T}\left\{F_{1}\left(a_{3}, \tau\right)-F_{1}\left(a_{4}, \tau\right)\right\}\right] & \text { for } \operatorname{Pr}=1\end{cases} \\
& u_{1}(\eta, \tau)=\left\{\begin{array}{l}
\lambda \sum_{n=0}^{\infty}\left[F_{2}\left(a_{1}, M, \tau\right)-F_{2}\left(a_{2} M, \tau\right)\right] \\
+\frac{G r}{(P r-1) \beta} \sum_{n=0}^{\infty}\left[F_{3}\left(a_{1}, \beta, \tau\right)-F_{3}\left(a_{2}, \beta, \tau\right)+r_{T}\left\{F_{3}\left(a_{3}, \beta, \tau\right)-F_{3}\left(a_{4}, \beta, \tau\right)\right\}\right. \\
-\left\{F_{4}\left(a_{1} \sqrt{P r}, \beta, \tau\right)-F_{4}\left(a_{2} \sqrt{P r}, \beta, \tau\right)\right\}-r_{T}\left\{F_{4}\left(a_{3} \sqrt{P r}, \beta, \tau\right)-F_{4}\left(a_{4} \sqrt{P r}, \beta, \tau\right)\right\} \\
-\left\{F_{2}\left(a_{1}, M, \tau\right)-F_{2}\left(a_{2}, M, \tau\right)\right\}-r_{T}\left\{F_{2}\left(a_{3}, M, \tau\right)-F_{2}\left(a_{4}, M, \tau\right)\right\} \\
\left.+\left\{F_{1}\left(a_{1} \sqrt{P r}, \tau\right)-F_{1}\left(a_{2} \sqrt{P r}, \tau\right)\right\}+r_{T}\left\{F_{1}\left(a_{3} \sqrt{P r}, \tau\right)-F_{1}\left(a_{4} \sqrt{P r}, \tau\right)\right\}\right] \\
\lambda \sum_{n=0}^{\infty}\left[F_{2}\left(a_{1}, M, \tau\right)-F_{2}\left(a_{2}, M, \tau\right)\right]-\frac{G r}{M^{2}} \sum_{n=0}^{\infty}\left[F_{2}\left(a_{1}, M, \tau\right)-F_{2}\left(a_{2}, M, \tau\right)\right. \\
+r_{T}\left\{F_{2}\left(a_{3}, M, \tau\right)-F_{2}\left(a_{4}, M, \tau\right)\right\} \\
\left.-\left\{F_{1}\left(a_{1}, \tau\right)-F_{1}\left(a_{2}, \tau\right)\right\}-r_{T}\left\{F_{1}\left(a_{3}, \tau\right)-F_{1}\left(a_{4}, \tau\right)\right\}\right]
\end{array}\right.
\end{aligned}
$$

where

$$
\begin{aligned}
& a_{1}=2 n+\eta, a_{2}=2 n+2 \eta, q=2 n+1 \eta, q=2 n+1 \eta \neq \\
& F_{1}(x, \tau)=\operatorname{erf}\left(\frac{x}{2 \sqrt{\tau}}\right)
\end{aligned}
$$

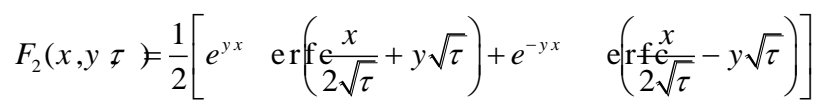




$$
\begin{aligned}
& F_{3}(x, y \mathcal{F})=\frac{1}{2} e^{\tau}\left[e^{\sqrt[x]{M^{2}+y}} \operatorname{er}\left(\frac{x}{2 \sqrt{\tau}}+\sqrt{\mathbb{R}^{2} 1+g}\right)\right. \\
& \left.+e^{-x \sqrt{M^{2}+y}} \operatorname{erf}\left(\frac{x}{2 \sqrt{\tau}}-\sqrt{M^{2}+y \tau}\right)\right]
\end{aligned}
$$

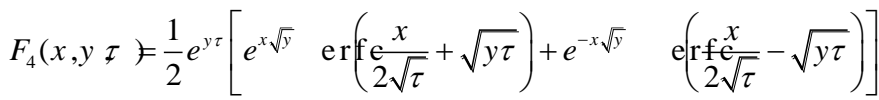

With the help of residue theorem, the steady state solutions $(\tau \rightarrow \infty)$ for the temperature and velocity distributions are given by

$$
\begin{aligned}
\theta(\eta) & =(1-\eta)+r_{T} \eta \\
u_{1}(\eta) & =\frac{\lambda \sinh M(+\eta)}{\sinh M}+\frac{G r}{M^{2}}\left[\left\{\left(1-\eta+r_{T} \eta\right\}\right.\right. \\
& \left.-\frac{\mathrm{s} \mathrm{i} \mathrm{nMh-( \eta 1+ \not )}}{\mathrm{s} \mathrm{i} \mathrm{nMh}}\right] \cdot \mathrm{r}
\end{aligned}
$$

In the absence of magnetic field $\left(M^{2}=0\right)$ the steady state solution for velocity distributions is given by

$$
u_{1}(\eta)=\frac{G r}{6}\left[\left(1-r_{T}\right) \eta^{3}-3 \eta^{2}+\left(2+r_{T}\right) \eta\right]
$$

Equations (17) and (19) are identical with the equations (2.12) and (2.13) obtained by Singh and Paul (2006).

\section{RESULTS AND DISCUSSION}

We have presented the non-dimensional fluid velocity and fluid temperature distributions for several values of magnetic parameter $M^{2}$, Prandtl number $\mathrm{Pr}$, Grashof number $\mathrm{Gr}$, wall temperature difference ratio $r_{T}$ and time $\tau$ in Figs.2-9.

Figs.2-6 represent the velocity $u_{1}$ against $\eta$ for several values of $M^{2}, P r, G r, r_{T}$ and $\tau$. It is seen from Fig.2 that the fluid velocity $u_{1}$ decreases for both the stationary wall as well as for the impulsive motion of one of the walls with an increase in magnetic parameter $M^{2}$. The presence of a magnetic field normal to the flow in an electrically conducting fluid introduces a Lorentz force which acts against the flow. This resistive force tends to slow down the flow and hence the fluid velocity decreases with the increase of the magnetic field parameter as observed in Fig.2. This trend is consistent with many classical studies on magneto-convection flow. Fig. 3 shows that the fluid velocity $u_{1}$ decreases for both the stationary wall as well as for the impulsive motion of one of the walls with an increase in Prandtl number $\mathrm{Pr}$. Physically, this is true because the increase in the Prandtl number is due to increase in the viscosity of the fluid which makes the fluid thick and hence causes a decrease in the velocity of the fluid. It is observed from Fig. 4 that an increase in Grashof number $G r$, leads to rise in the values of fluid velocity $u_{1}$ for both the stationary wall as well as for the impulsive motion of one of the walls due to enhancement in buoyancy force. Figs. 5 and 6 show that the fluid velocity $u_{1}$ increases for both the stationary wall as well as for the impulsive motion of one of the walls with an increase in either temperature difference ratio $r_{T}$ or time $\tau$. It is seen from Fig. 7 that the fluid temperature $\theta$ decreases with an increase in Prandtl number $\mathrm{Pr}$. The effect of the Prandtl number is very important in the temperature field. A fall in fluid temperature occurs due to an increasing value of the Prandtl number. This is in agreement with the physical fact that the thermal boundary layer thickness decreases with an increase in $P r$. It is observed from Figs. 8 and 9 that the fluid temperature $\theta$ increases with an increase in in either $r_{T}$ or time $\tau$.

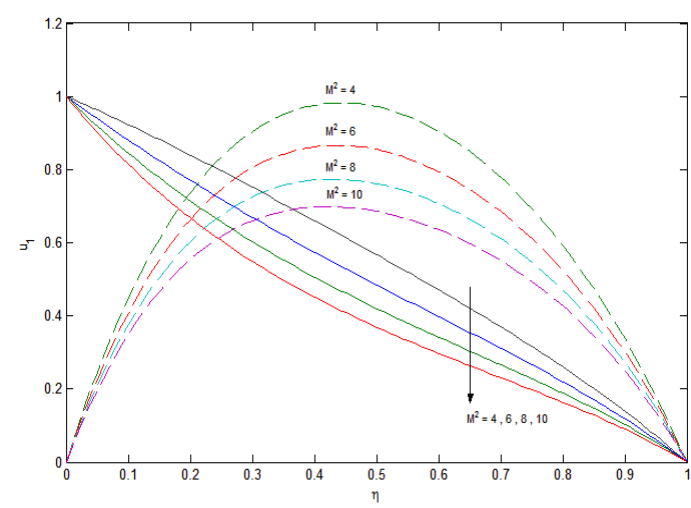

Figure 2: Velocity for the variation of $M^{2}$ when $\operatorname{Pr}=3$, $G r=5, r_{T}=0.5$ and $\tau=0.5:-----$ for the stationary wall $(\lambda=0)$ and when one of the walls starts impulsively $(\lambda=1)$.

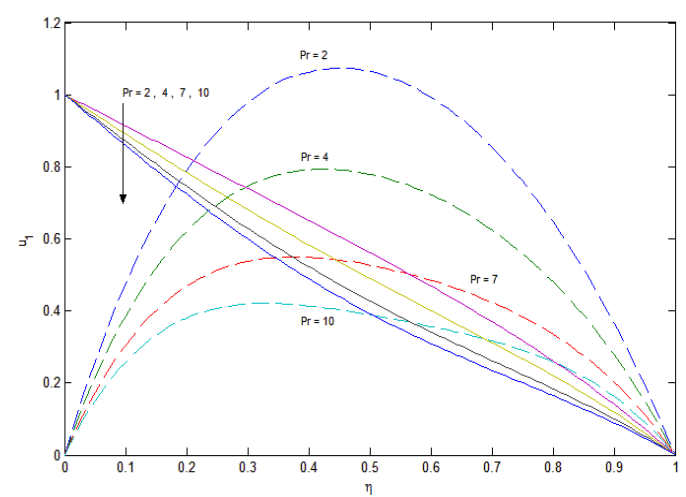

Figure 3: Velocity for the variation of $\operatorname{Pr}$ when $M^{2}=5$, $G r=5, r_{T}=0.5$ and $\tau=0.5:-----$ for the stationary wall $(\lambda=0)$ and $\longrightarrow$ when one of the walls starts impulsively $(\lambda=1)$. 


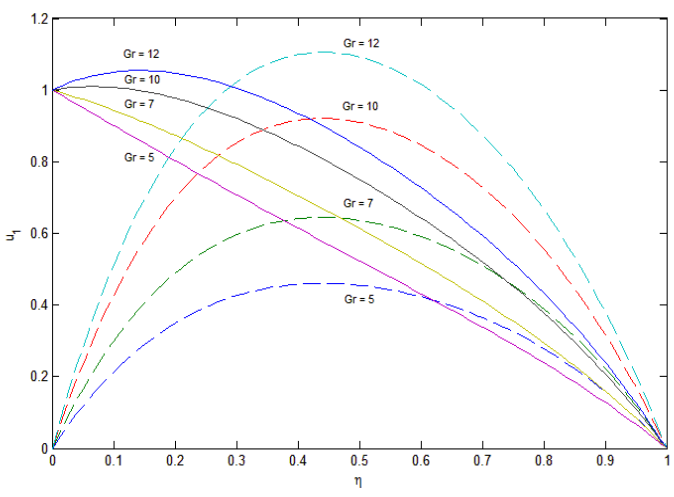

Figure 4: Velocity for the variation of $G r$ when $M^{2}=5$, $\operatorname{Pr}=3, r_{T}=0.5$ and $\tau=0.5:-----$ for the stationary wall $(\lambda=0)$ and when one of the walls starts impulsively $(\lambda=1)$.

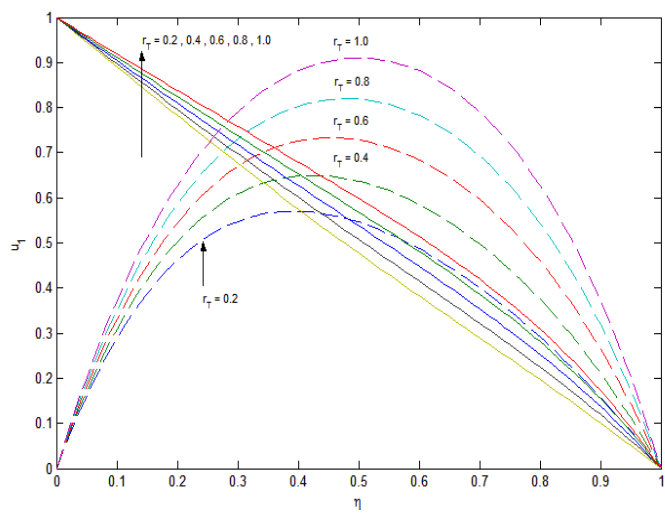

Figure 5: Velocity for the variation of $r_{T}$ when $M^{2}=5$, $P r=3, G r=5$ and $\tau=0.5:-----$ for the stationary wall $(\lambda=0)$ and when one of the walls starts impulsively $(\lambda=1)$.

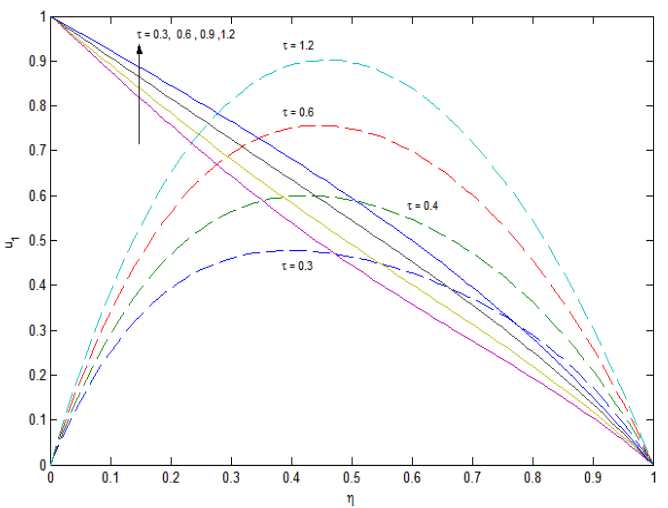

Figure 6: Velocity for the variation of $\tau$ when $M^{2}=5$, $P r=3, G r=5$ and $r_{T}=0.5$ : for the stationary wall $(\lambda=0)$ and when one of the walls starts impulsively $(\lambda=1)$.

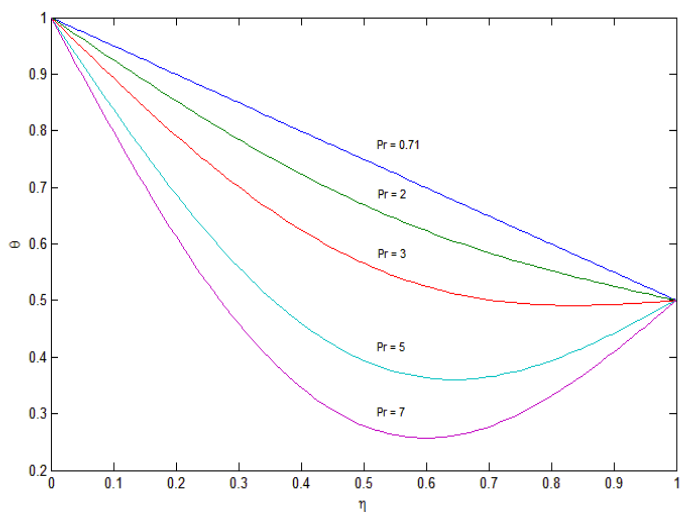

Figure 7: Temperature for the variation of $P r$ when $r_{T}=0.5$ and $\tau=0.5$.

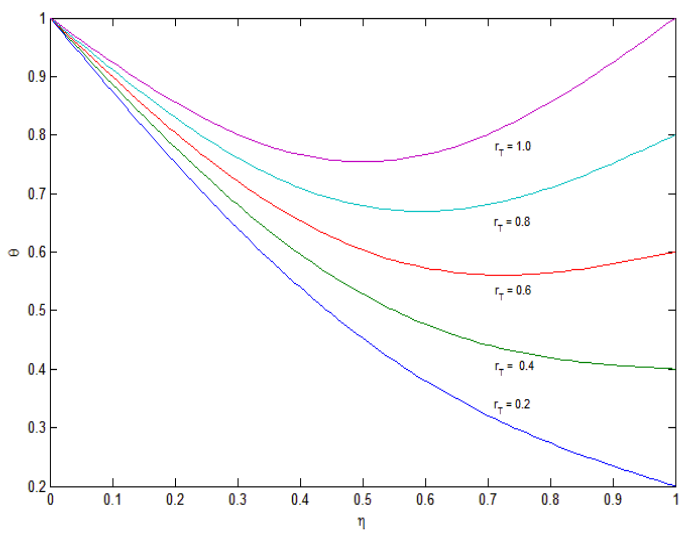

Figure 8: Temperature for the variation of $r_{T}$ when $\mathrm{Pr}=3$ and $\tau=0.5$.

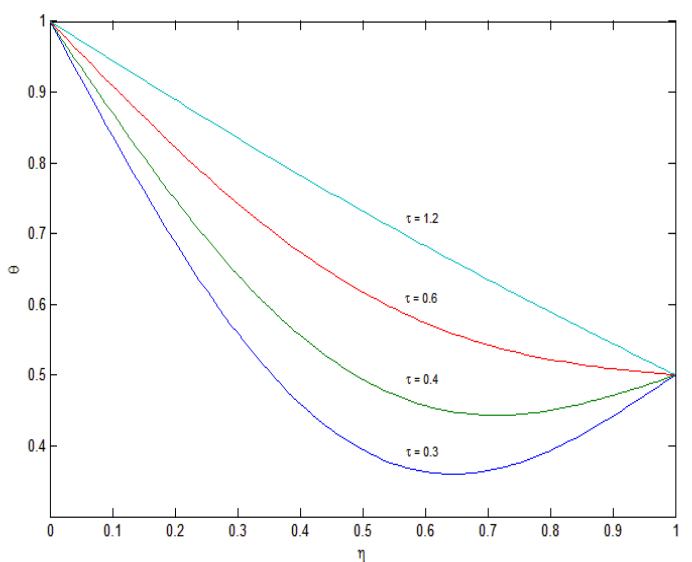

Figure 9: Temperature for the variation of $\tau$ when $P r=3$ and $r_{T}=0.5$.

The rate of heat transfer $-\theta^{\prime}(0)$ at the wall $(\eta=0)$ is given by 


$$
-\theta^{\prime}(0)= \begin{cases}\sum_{0}^{\infty}\left[H_{1}(2 n \sqrt{\operatorname{Pr}}, \tau)\right. & \\ +H_{1}((2 n+2) \sqrt{\operatorname{Pr}}, \tau) & \text { for } \operatorname{Pr} \neq 1 \\ \left.-2 r_{T} H_{1}((2 n+1) \sqrt{\operatorname{Pr}}, \tau)\right] & \\ \sum_{0}^{\infty}\left[H_{1}(2 n, \tau)+H_{1}(2 n+2, \tau)\right. & \text { for } \operatorname{Pr}=1, \\ \left.-2 r_{T} H_{1}(2 n+1, \tau)\right] & \end{cases}
$$

where

$$
H_{1}(x, \tau)=\frac{1}{\sqrt{\pi \tau}} e^{-\frac{x^{2}}{4 \tau}}
$$

Numerical results of the rate of heat transfer $-\theta^{\prime}(0)$ at the wall $(\eta=0)$ against the temperature difference ratio $r_{T}$ are presented in the Table-I for several values of Prandtl number $\operatorname{Pr}$ and time $\tau$. Table-I shows that the rate of heat transfer $-\theta^{\prime}(0)$ increases with an increase in Prandtl number $P r$ while it decreases with an increase in time $\tau$ for the fixed values of $r_{T}$. Further, it is seen that for fixed value of $P r$ and $\tau$, the rate of heat transfer $-\theta^{\prime}(0)$ decreases with an increase in temperature difference ratio $r_{T}$.

Table-I. Rate of heat transfer $-\theta^{\prime}(0)$ at the wall $(\eta=0)$

\begin{tabular}{|c|l|l|l|l|l|l|l|c|}
\hline & \multicolumn{9}{|l|}{$\operatorname{Pr}$} & \multicolumn{1}{l|}{$\tau$} \\
\hline$r_{T}$ & 2 & 4 & 7 & 10 & 0.3 & 0.6 & 0.9 & 1.2 \\
\hline 0.00 & 1.16971 & 1.59684 & 2.11101 & 2.52313 & 1.78427 & 1.27866 & 1.10356 & 1.03859 \\
0.25 & 0.96209 & 1.48886 & 2.07913 & 2.51463 & 1.71106 & 1.09787 & 0.87944 & 0.79824 \\
0.50 & 0.75447 & 1.38088 & 2.04726 & 2.50613 & 1.63784 & 0.91711 & 0.65533 & 0.55789 \\
0.75 & 0.54684 & 1.27290 & 2.01539 & 2.49763 & 1.56461 & 0.73638 & 0.43121 & 0.31754 \\
1.00 & 0.33922 & 1.16491 & 1.98351 & 2.48913 & 1.49139 & 0.55564 & 0.20709 & 0.07719 \\
\hline
\end{tabular}

The non-dimensional shear stress $\tau_{x}\left[=\left(\frac{\partial u_{1}}{\partial \eta}\right)_{\eta=0}\right]$ at the wall $(\eta=0)$ is give by

$$
\begin{aligned}
& \int \lambda \sum_{n=0}^{\infty}\left[H_{2}(2 n, M, \tau)+H_{2}(2 n+2, M, \tau)\right] \\
& +\frac{G r}{(P r-1) \beta} \sum_{n=0}^{\infty}\left[H_{3}(2 n, \beta, \tau)+H_{3}(2 n+2, \beta, \tau)\right. \\
& -2 r_{T}\left\{H_{3}(2 n+1, \beta, \tau)-H_{4}((2 n+1) \sqrt{P r}, \beta, \tau)\right\} \\
& -\left\{H_{4}(2 n \sqrt{P r}, \beta, \tau)+H_{4}((2 n+2) \sqrt{P r}, \beta, \tau)\right\} \\
& -\left\{H_{2}(2 n, M, \tau)+H_{2}(2 n+2, M, \tau)\right\} \\
& \tau_{x}=\left\{+2 r_{T}\left\{H_{2}(2 n+1, M, \tau)+H_{1}((2 n+1) \sqrt{P r}, \tau)\right\}\right. \\
& \left.-\left\{H_{1}(2 n \sqrt{P r}, \tau)+H_{1}((2 n+2) \sqrt{P r}, \tau)\right\}\right] \quad \text { for } \operatorname{Pr} \neq 1 \\
& \lambda \sum_{n=0}^{\infty}\left[H_{2}(2 n, M, \tau)+H_{2}(2 n+2, M, \tau)\right] \\
& -\frac{G r}{M^{2}} \sum_{n=0}^{\infty}\left[H_{2}(2 n, M, \tau)+H_{2}(2 n+2, M, \tau)\right. \\
& \left\{\begin{array}{l}
-2 r_{T}\left\{H_{2}(2 n+1, M, \tau)+H_{1}(2 n+1, M, \tau)\right\} \\
\left.+\left\{H_{1}(2 n, \tau)+H_{1}(2 n+2, \tau)\right\}\right]
\end{array}\right.
\end{aligned}
$$

where

$H_{2}(x, y, \tau)=\frac{1}{2}\left[y\left\{e^{y x} \operatorname{erfc}\left(\frac{x}{2 \sqrt{\tau}}+y \sqrt{\tau}\right)-e^{-y x} \operatorname{erfc}\left(\frac{x}{2 \sqrt{\tau}}-y \sqrt{\tau}\right)\right\}\right.$

$$
\begin{gathered}
\left.-\frac{2}{\sqrt{\pi \tau}} e^{-\left(\frac{x^{2}}{4 \tau}+y^{2} \tau\right)}\right], \\
H_{3}(x, y, \tau)=\frac{1}{2} e^{y \tau}\left[\sqrt { M ^ { 2 } + y } \left\{e^{x \sqrt{M^{2}+y}} \operatorname{erfc}\left(\frac{x}{2 \sqrt{\tau}}+\sqrt{\left(M^{2}+y\right) \tau}\right)\right.\right. \\
\left.\left.\left.-e^{-x \sqrt{M^{2}+y}} \operatorname{erfc}\left(\frac{x}{2 \sqrt{\tau}}-\sqrt{\left(M^{2}+y\right) \tau}\right)\right\}-\frac{2}{\sqrt{\pi \tau}} e^{-\left\{\frac{x^{2}}{4 \tau}+\left(M^{2}+y\right) \tau\right.}\right\}\right] \\
H_{4}(x, y, \tau)= \\
-\frac{1}{2} e^{y \tau}\left[\sqrt { y } \left\{e^{x \sqrt{y}} \operatorname{erfc}\left(\frac{x}{2 \sqrt{\tau}}+\sqrt{y \tau}\right)\right.\right. \\
\left.\left.\left.-e^{-x \sqrt{y}} \operatorname{erf}\left(\frac{x}{2 \sqrt{\tau}}-\sqrt{y \tau}\right)\right\}-\frac{2}{\sqrt{\pi \tau}} e^{-\left(\frac{x^{2}}{4 \tau}+y \tau\right.}\right)\right]
\end{gathered}
$$

Numerical results of the non-dimensional shear stress at the wall $(\eta=0)$ are presented in Figs.10-13 against magnetic parameter $M^{2}$ for several values of Prandtl number $\mathrm{Pr}$, Grashof number $G r$, temperature difference ratio $r_{T}$ and time $\tau$. Fig.10 displays that the shear stress $\tau_{x}$ decreases for both stationary wall as well as for the impulsive motion of one of the walls with an increase in either Prandtl number $P r$ or magnetic parameter $M^{2}$. Physically, it is possible because fluids with Prandtl numbers move slowly and hence there is more friction at the walls. Figs.11-13 show that the shear stress $\tau_{x}$ increases for both the stationary wall as well as for the impulsive motion of one of the walls with an increase in either Grashof number $\mathrm{Gr}$ or temperature difference ratio $r_{T}$ or time $\tau$. These results are in agrement with the fact that the velocity increases with an 
increase in either $G r$ or $r_{T}$ or $\tau$ while it decreases with an increase in either $M^{2}$ or $\mathrm{Pr}$. Further, it is observed from Figs.10-13 that the shear stress $\tau_{x}$ at the wall $\eta=0$ in case of impulsive motion of one of the walls is less than that of the stationary wall.

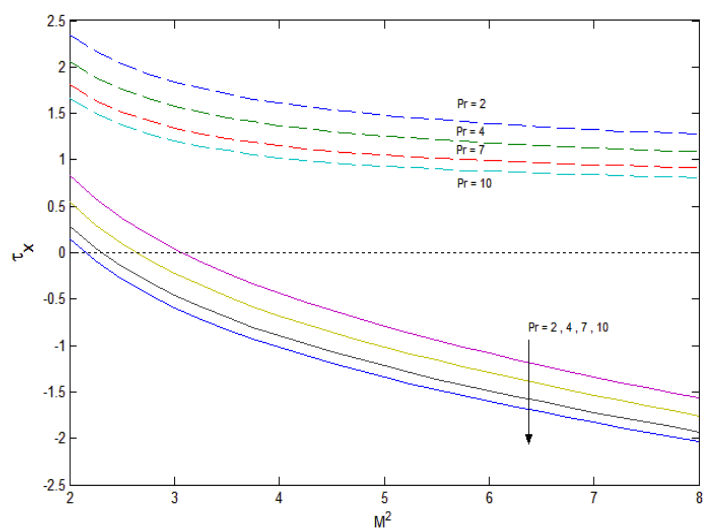

Figure 10: Shear stress $\tau_{x}$ for the variation of $\operatorname{Pr}$ when $G r=5, r_{T}=0.5$ and $\tau=0.5:-----$ for the stationary wall $(\lambda=0)$ and when one of the walls starts impulsively $(\lambda=1)$.

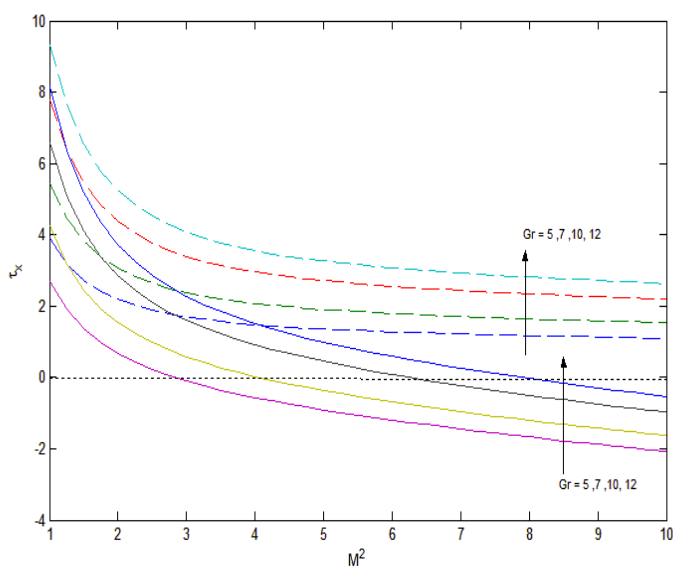

Figure 11: Shear stress $\tau_{x}$ for the variation of $G r$ when $\operatorname{Pr}=3, r_{T}=0.5$ and $\tau=0.5:-----$ for the stationary wall $(\lambda=0)$ and when one of the walls starts impulsively $(\lambda=1)$.

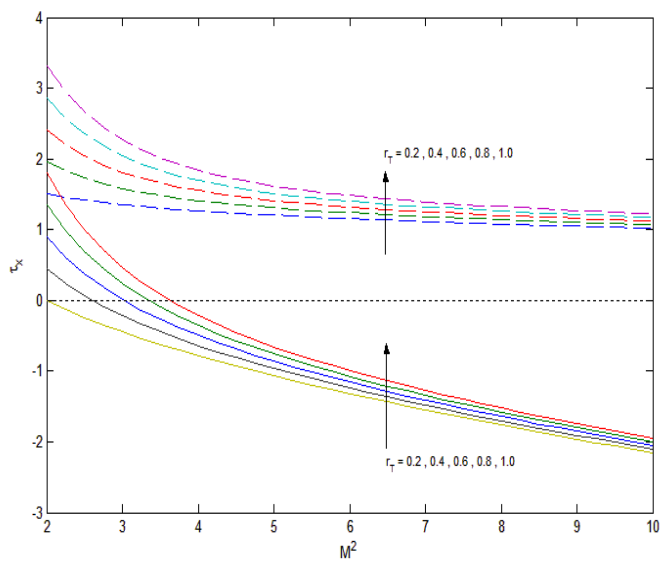

Figure 12: Shear stress $\tau_{x}$ for the variation of $r_{T}$ when $\operatorname{Pr}=3, G r=5$ and $\overline{\tau=0.5:---}--$ for the stationary wall $(\lambda=0)$ and when one of the walls starts impulsively $(\lambda=1)$.

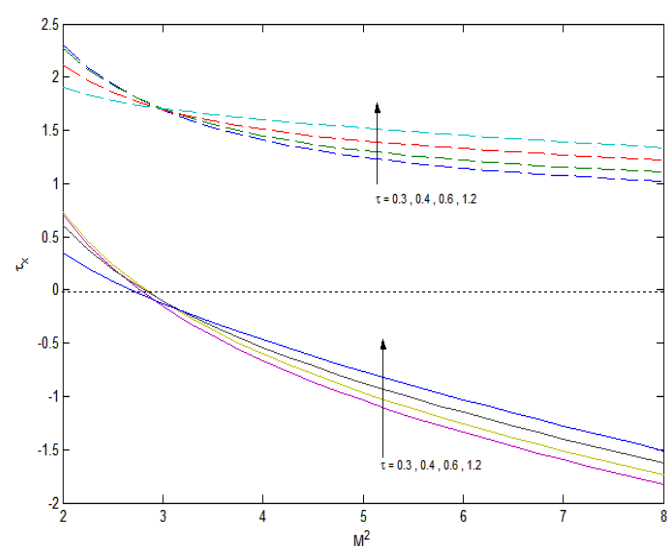

Figure 13: Shear stress $\tau_{x}$ for the variation of $\tau$ when $\operatorname{Pr}=3, G r=5$ and $r_{T}=0.5:-----$ for the stationary wall $(\lambda=0)$ and when one of the walls starts impulsively $(\lambda=1)$.

\section{CONCLUSION}

We have studied the transient MHD natural convection flow of a viscous incompressible electrically conducting fluid confined between two infinitely long vertical walls heated/cooled asymmetrically. It is observed that magnetic field has a retarding influence whereas buoyancy force has an accelerating influence on the fluid velocity $u_{1}$ for both stationary wall as well as for impulsive motion of one of the walls. It is seen that the effect of the Prandtl number $P r$ is very important in the temperature field. An increase in fluid temperature $\theta$ occurs due to an increase in time. Further, the shear stress $\tau_{x}$ at the wall $\eta=0$ increases for both the stationary wall as well as for impulsive motion of one of the walls with an increase in either Grashof number $G r$ or temperature difference ratio $r_{T}$ or time $\tau$. Prandtl number $P r$ tends to enhance the rate of heat transfer $-\theta^{\prime}(0)$ while temperature difference ratio $r_{T}$ as well as time $\tau$ tend to reduce the rate of heat transfer $-\theta^{\prime}(0)$ at the wall $(\eta=0)$. 


\section{REFERENCES}

[1] Singer, R. M. (1965). Transient magnetohydrodynamic flow and heat transfer. Zeitschrift für Angewandte Mathematik und Mechanik. 16(4): 483-494.

[2] Joshi, H. M. (1988). Transient effects in natural convection cooling of vertical parallel plates. Int. Comm. Heat Mass Transfer. 15: 227-238.

[3] Singh, A. K. (1988). Natural convection in unsteady Couette motion. Defense Science Journal. 38(1): 35-41.

[4] Sacheti, N. C., Chandran, P. and Singh, A. K. (1994) An exact solution for unsteady magnetohydrodynamic free convection flow with constant heat flux. Int. Comm. Heat and Mass Transfer. 21(1): 131-142.

[5] Singh, A. K., Gholami, H. R. and Soundalgekar, V. M (1996). Transient free convection flow between two vertical parallel plates. Heat and Mass Transfer. 31: 329-331.

[6] Jha, B. K. (2001). Natural Convection in unsteady MHD Couette flow. Heat and Mass Transfer. 37: 329-331.

[7] Chamkha, A. J. (2001). Unsteady laminar hydromagnetic flow and heat transfer in porous channels with temperature-dependent properties. Int.J. Num. Meth.Heat and Fluid Flow. 11(5-6): 430-448.

[8] Jha, B. K., Singh, A. K. and Takhar, H. S. (2003) Transient free convection flow in a vertical channel due to symmetric heating. Int. J. Appl. Mech. Eng. 8(3): 497-502.

[9] Singh, A. K. and Paul, T. (2006). Transient natural convection between two vertical walls heated/cooled asymmetrically. Int. J. Appl. Mech. Eng. 11(1): 143-154.

[10] Grosan, T. and Pop, I. (2007). Thermal radiation effect on fully develop mixed convection flow in a vertical channel. Technische Mechanik. 27(1): 37-47.
[11] Narahari, M. Natural convection in unsteady Couette flow between two vertical parallel plates in the presence of constant heat flux and radiation. MACMESE'09 Proceedings of the 11th WSEAS international conference on Mathematical and computational methods in science and engineering.

[12] Narahari, M. (2009). Oscillatory plate temperature effects of free convection flow of dissipative fluid between long vertical parallel plates. Int. J. of Appl. Math and Mech. 5(3): $30-46$

[13] Narahari, M. (2010). Effects of thermal radiation and free convection currents on the unsteady couette flow between two vertical parallel plates with constant heat flux at one boundary. WSEAS Transactions on Heat and Mass Transfer. 5(1): 21-30.

[14] Rajput, U.S. and Sahu, P. K. (2011). Transient free convection MHD flow between two long vertical parallel plates with constant temperature and variable mass diffusion. Int. J. Math. Analysis. 5(34): 1665-6671.

[15] Das, S., Sarkar, B. C. and Jana, R. N. (2012). Radiation effects on free convection MHD Couette flow started exponentially with variable wall temperature in presence of heat generation. Open J. Fluid Dynamics. 2: $14-27$

[16] Mandal, C., Das, S. and Jana, R. N. (2012). Effect of radiation on transient natural convection flow between two vertical walls. Int. J. Appl. Inf. Systems. 2(2): 49-56.

[17] Ahmed, N., Sarma, K. and Barua, D. P. (2012) Magnetic field effect on free convective oscillatory flow between two vertical parallel plates with periodic plate temperature and dissipative heat. Appl. Math. Sc. 6(39): 1913-1924.

[18] Sarkar, B. C., Das, S. and Jana, R. N. (2012). Effects of radiation on MHD free convective Couette flow in a rotating system. Int. J. Eng. Res. and Appl. 2(4): 2346-2359. 